\title{
Spatial spectral and energy efficiencies of cellular networks limited by co-channel interference and path loss in Nakagami- $m$ fading environment
}

\author{
Abdulbaset M. Hamed ${ }^{*}$ (D) and Raveendra K. Rao
}

\begin{abstract}
A comprehensive framework for design of hexagonal cellular network system in terms of spatial spectral and energy efficiencies is presented. The communication environment in the system is assumed to be Nakagami-m fading coupled with simplified path loss model and co-channel interference. Three base station antenna configurations namely, omni, $120^{\circ}$ and $60^{\circ}$ are considered. Closed-form expressions for spatial spectral and energy efficiencies and coverage probability are derived and illustrated as a function of signal-to-noise ratio, cell radius, reuse distance, path loss exponent factor, and fading figure. A discussion of trade-offs among spatial spectral efficiency, spatial energy efficiency, and coverage probability as a function of parameters of cellular system and propagation environment is also provided. Numerical results show that the use of sectorized antenna system in the cellular network provides significant improvements even under severe propagation and interference conditions. For example, it is observed that the $120^{\circ}$ base station antenna configuration system provides nearly double the spatial energy efficiency relative to the omni configuration system.
\end{abstract}

Keywords: Cellular networks, Energy efficiency, Fading channel, Co-channel interference, Spectral efficiency, Generalized area, Coverage probability

\section{Introduction}

The ever increasing demand for a variety of mobile communication services has resulted in the expansion of existing cellular network infrastructure. This has caused a huge increase in the energy consumption, particularly at the base stations (BSs) in the network. The BSs account for nearly $18 \%$ of the total energy consumption in the network, and a significant amount of it is used for signal transmission [1]. The escalation of energy consumption due to the network expansion results in a corresponding increase in the emission of greenhouse gases [2]. Also, studies have shown an exponential growth in electrical energy consumption by the users of the network [3, 4]. It is predicated that an annual increase in electrical energy consumption of nearly $20 \%$ is a conservative estimate for

*Correspondence: ahamed6@uwo.ca

Faculty of Engineering, Department of Electrical and Computer Engineering, Western University, London, Ontario, N6A 5B9, Canada cellular networks. In order to minimize the effect of global warming and sustainable growth, government agencies are imposing strict limits on greenhouse gas emissions. This has necessitated in the design and implementation of cellular networks that are energy efficient. Towards this goal, European Commission has initiated FP7 projects that include EARTH and ECONET [5]. Until recently researches have used spectral efficiency (SE) as a metric for optimum design of networks and did not pay attention to the energy efficiency (EE) of the network. However, due to the limits imposed on greenhouse gases, it has become important to optimize EE metric for efficient design of cellular networks. Optimizing one metric contradicts with the other, as there always exist a trade-off between EE and SE metrics [6] in any given network. The SE and EE bounds have been investigated over small- and large-scale fading channels using the Moment Generation Function (MGF) approach in [7]. These metrics have been examined using simulations over fading channels, and an 
adaptive technique is proposed to improve the metrics for a given quality of service $(\mathrm{QoS})$ in $[8,9]$. The trade-off between $\mathrm{SE}$ and $\mathrm{EE}$ for an interference limited wireless network using a power allocation algorithm is examined in [10]. The effect of shadowing and frequency reuse factor on $\mathrm{SE}$ and $\mathrm{EE}$ for a wireless network is considered in [11]. The SE and EE for a cellular network are examined for a simple interference model and Rayleigh fading environment in $[12,13]$. These two metrics have been examined for single- and multi-cell cellular networks subjected to co-channel interference in fading environment in [14]. However, the spatial characteristics of cellular networks are not considered. In a cellular network, it is important to consider the spatial EE and SE metrics. Area spectral efficiency has been introduced in [15] as a performance metric in a cellular network, and it depends on the cellular geometry and reuse distance. In [16], the trade-off between spatial spectral efficiency and spatial energy efficiency is examined for a cellular network. Optimization of these two metrics is examined for the macro-cell with BSs in pico-cells. Kim et al. [17] have used the same metrics to investigate the performance of a hierarchical cellular network. Zhang et al. [18] have introduced the notion of affected area to examine the spatial characteristics of cellular network. The coverage probability is examined as a function of spectral and energy efficiencies to analyze the performance of cellular networks in [19]. These efficiencies are related to the outage capacity and the coverage probability and are important performance metrics in the design of an energy-efficient cellular network. The effect of path loss and Nakagami- $m$ fading environment on the outage probability for interference-limited cellular network has been investigated in [20]. The analysis has been done by deriving statistical distributions of SNR and SINR for Poisson point process in a wireless network. To the best of our knowledge, spatial efficiency metrics have not been thoroughly investigated for efficient design of cellular network, particularly taking into account the statistical behavior of propagation environment modelled as Nakagami- $m$ distribution. Thus, the intent of this paper is to present a thorough investigation of spatial efficiency metrics and coverage probability of a cellular network as a function of path loss, co-channel interference, cell dimensions, and statistical parameters of wireless environment in the system.

The paper is organized as follows. Multi-cell hexagonal cellular network is described in Section 2. The system statistical models, signal-to-interference-plus-noise ratio model, and fading environment are also presented. Section 3 provides definitions of spectral and energy efficiencies for single- and multi-cell systems and closedform expressions for these metrics are derived and illustrated. Section 4 addresses the concept of affected area in a cellular system and an expression is derived for the affected area. Spatial spectral and energy efficiencies are also considered in the same section. In Section 5, coverage probability of multi-cell cellular system is considered taking into account the propagation environment and co-channel interference. Finally, the paper is concluded in Section 6.

\section{Cellular network and propagation environment}

A cellular network consisting of homogeneous macrocells with hexagonal tessellation is shown in Fig. 1. The base stations (BSs) are located at the center of each cell, and a user is assumed to be served by the closest BS. The power and bandwidth resources are assumed to be equally assigned to all cells in the network. The downlink, BS to user, efficiency analysis is of interest in this work. Three different BS antenna configuration are considered in this work: (i) omni, (ii) $120^{\circ}$, and (iii) $60^{\circ}$. Figure 1 shows an omni hexagonal cellular network with $R$, the cell radius; $D$, the frequency reuse distance; and $D_{j, i}$, the interfering distance. The blue cells in Fig. 1 use the same frequency band and hence are the co-channel cells.

\subsection{Propagation and interference models}

In the cellular network shown in Fig. 1, let the transmission power of $j^{\text {th }}$ BS be $P_{j}$ and the mobile user $i$ be served by the closest BS. Thus, the power received at the $i^{\text {th }}$ mobile user at a distance of $r_{j, i}$ from the $j^{\text {th }}$ BS can be written as

$$
P_{j, i}=P_{j} r_{j, i}^{-\alpha} h_{j, i}^{2},
$$

where $\alpha$ is the path-loss exponent, and $h_{j, i}$ is a random variable representing the channel fading coefficient between the user and the BS. The aggregated interference power at the $i^{t h}$ user due to co-channel cells can be expressed as

$$
I_{i}=\sum_{k=1}^{N_{I}} P_{k} D_{k, i}^{-\alpha} h_{k, i}^{2},
$$

where $D_{k, i}$ is the distance between $k^{\text {th }}$ interfering BS and $i^{t h}$ user, and $N_{I}$ the number of co-channel interfering cells. The generic expression for the instantaneous signal-tointerference-plus-noise ratio (SINR) at the $i^{\text {th }}$ user can be expressed as

$$
\gamma_{j, i}^{I}=\frac{P_{j} r_{j, i}^{-\alpha} h_{j, i}^{2}}{N_{o} B+\sum_{\substack{k=1 \\ k \neq j}}^{N_{I}} P_{k} D_{k, i}^{-\alpha} h_{k, i}^{2}},
$$

where $N_{o}$ is the power spectral density of Additive White Gaussian Noise (AWGN), and $B$ is the bandwidth of the user. The resulting SINR is a random variable in which $h^{2}$ represents the power associated with the fading coefficient of the channel. 


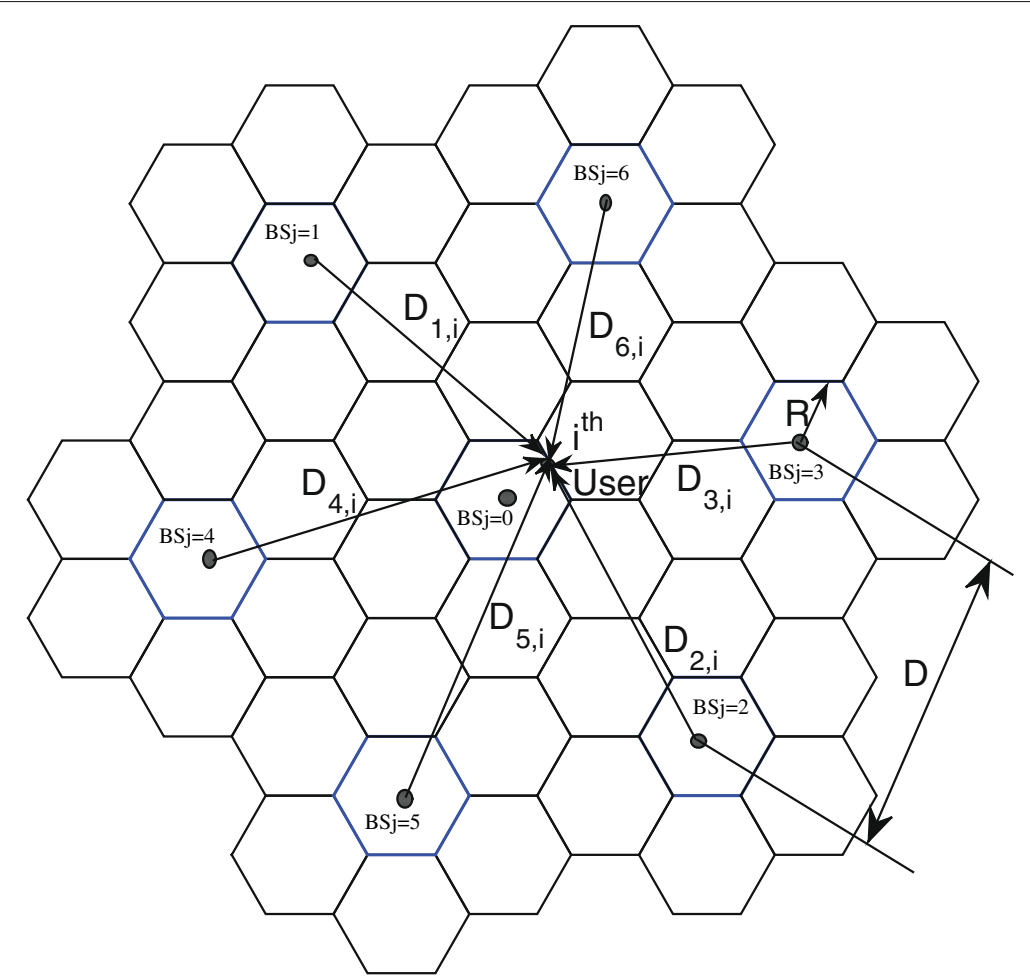

Fig. 1 Hexagonal cellular network. $D$ reuse distance, $R$ cell radius, and $D_{j, i}$ interfering distance

\subsection{User's location model}

It is assumed that the users are randomly distributed across a given cell; hence, it is important to model the users' locations in the system. The BS coverage area can be approximated to that of a circle with radius $R$. The users' locations can be defined using the polar coordinates as:

$$
\mathcal{L}(r, \theta)=r e^{j \theta}
$$

where $r$ is the distance from the BS and $\theta$ is the angle with respect to a certain reference. The probability density functions (pdf) for $r$ and $\theta$ are given by [21]

$$
\begin{aligned}
& f_{r}(r)=\frac{2 r}{R^{2}}, \quad 0 \leqslant r \leqslant R \\
& f_{\theta}(\theta)=\frac{1}{2 \pi}, \quad 0 \leqslant \theta \leqslant 2 \pi
\end{aligned}
$$

Using (5) and (6), the user's location can be estimated and hence the path-loss in (1).

\subsection{Channel model}

Wireless channel in the network suffers from various impairments and effects, including small-scale fading, which is due to multi-path effect that causes serious degradation of signal-to-noise ratio (SNR) leading to poor system performance. The received powers in (1) and (2) are randomly scaled by the factor $h^{2}$. Many distributions have been used to model the multi-path fading coefficient $h$. In this paper, Nakagami- $m$ fading model is used to model $h$ and the pdf of $h^{2} \sim \mathcal{G}(m, \Omega)$ is given by

$$
f_{\gamma_{j, i}}\left(\gamma_{j, i}\right)=\frac{m^{m} \gamma_{j, i}^{m-1}}{\bar{\gamma}_{j, i}^{m} \Gamma(m)} \exp \left(-\frac{m \gamma_{j, i}}{\bar{\gamma}_{j, i}}\right), \quad \gamma_{j, i} \geqslant 0
$$

where $m$ is the fading figure, $\Omega=\overline{h^{2}} / m, \gamma_{j, i}=\frac{P_{j, i} h_{j, i}^{2}}{N_{o} B}$ is the instantaneous SNR, $\bar{\gamma}_{j, i}=\frac{P_{j, i} \bar{h}_{j}{ }_{j, i}}{N_{o} B}$ is the average SNR, and $P_{j, i}=P_{j} r_{j, i}^{-\alpha}$ is the $i^{\text {th }}$ user's received power due to path loss at a distance $r_{j, i}$ from the $j^{\text {th }} \mathrm{BS}$, and hence, the average SNR is $\bar{\gamma}_{j, i} r^{-\alpha}$.

\section{Energy and spectral efficiencies of single- and multi-cellular system}

Bandwidth and power are the two main resources of concern in cellular network planning. Since the demand for high data rates is growing rapidly, SE-oriented cellular network design causes a large increase in energy consumption due to high QoS requirement. The next generation cellular networks therefore should be planned not only for high spectral efficiency but also for high energy efficiency. In this section, the downlink energy and spectral efficiencies are studied considering multi-path fading, co-channel interference, and frequency reuse factor for single- and multi-cell scenarios. 


\subsection{Analysis of single-cell scenario}

In this section, the energy and the spectral efficiency metrics for a single-cell scenario with cell radius $R$ as shown in Fig. 2 are examined. The BS serves a set of $K$ users in which the power and bandwidth resources are equally shared among all the users. Thus, each user occupies $B$ $\mathrm{Hz}$ of bandwidth and receives power $P_{i}$. The users are randomly located as described by (4).

\subsubsection{Spectral efficiency}

The downlink spectral efficiency quantifies the delivered data rate to the users over the bandwidth. In the case of a fading channel where the random fading coefficients are independent, the aggregated downlink spectral efficiency can be expressed as

$$
\eta_{s}^{d}=\sum_{i=1}^{K} \mathbb{E}\left[\log _{2}\left(1+\frac{P_{i} h_{i}^{2}}{N_{o} B} r_{i}^{-\alpha}\right) \mid h_{i}^{2}\right], \quad(b i t / s / H z)
$$

where $K$ is the total number of users in the cell and the expectation, $\mathbb{E}$, is taken over $h_{j, i}^{2}$. In this scenario, since there is only one BS; the subscript $j$ notation is dropped in the analysis. The downlink received power is $P_{i}^{p l}=$
$P_{i} r_{i}^{-\alpha}$ and hence $\gamma_{i}^{p l}=\gamma_{i} r_{i}^{-\alpha}$. Spectral efficiency can also be obtained by averaging over the fading channel distribution as

$$
\eta_{s}^{d}=\int_{0}^{\infty} \sum_{i=1}^{K}\left[\log _{2}\left(1+\gamma_{i}\right) \mid \gamma_{i}\right] f_{\gamma_{i}}\left(\gamma_{i}\right) d \gamma_{i}
$$

By substituting the pdf of $\gamma_{i}$, (7), in (9) and using ([22] Eq. (3.383.10), Eq. (3.326.2)), we get

$$
\begin{aligned}
\eta_{s}^{d}\left(r_{i}, \alpha\right)= & \frac{1}{\log 2} \sum_{i=1}^{K} \sum_{k=1}^{m} e^{\frac{m}{\bar{\gamma}_{i}} r_{i}^{\alpha}} \\
& \times \Gamma\left(-m+k, \frac{m}{\bar{\gamma}_{i}} r_{i}^{\alpha}\right)\left(\frac{\bar{\gamma}_{i}}{m} r_{i}^{-\alpha}\right)^{k-m}
\end{aligned}
$$

where $\Gamma(\beta, x)=\frac{1}{\Gamma(\beta)} \int_{x}^{+\infty} t^{\beta-1} e^{-t} d t$ is the complementary incomplete gamma function. The spectral efficiency is a function of average SNR, fading figure $m$, and the path loss exponent. Since the user's distance $r_{i}$ is random, (10) needs to be averaged over the density of $r_{i}$. By expressing the incomplete gamma function term $\Gamma\left(-m+k, \frac{m}{\bar{\gamma}_{j, i}} r_{i}^{\alpha}\right)$ using ([23], Eq. (06.06.03.0009.01)) and using (5) in (10), the downlink spectral efficiency can be obtained in closed-form as:

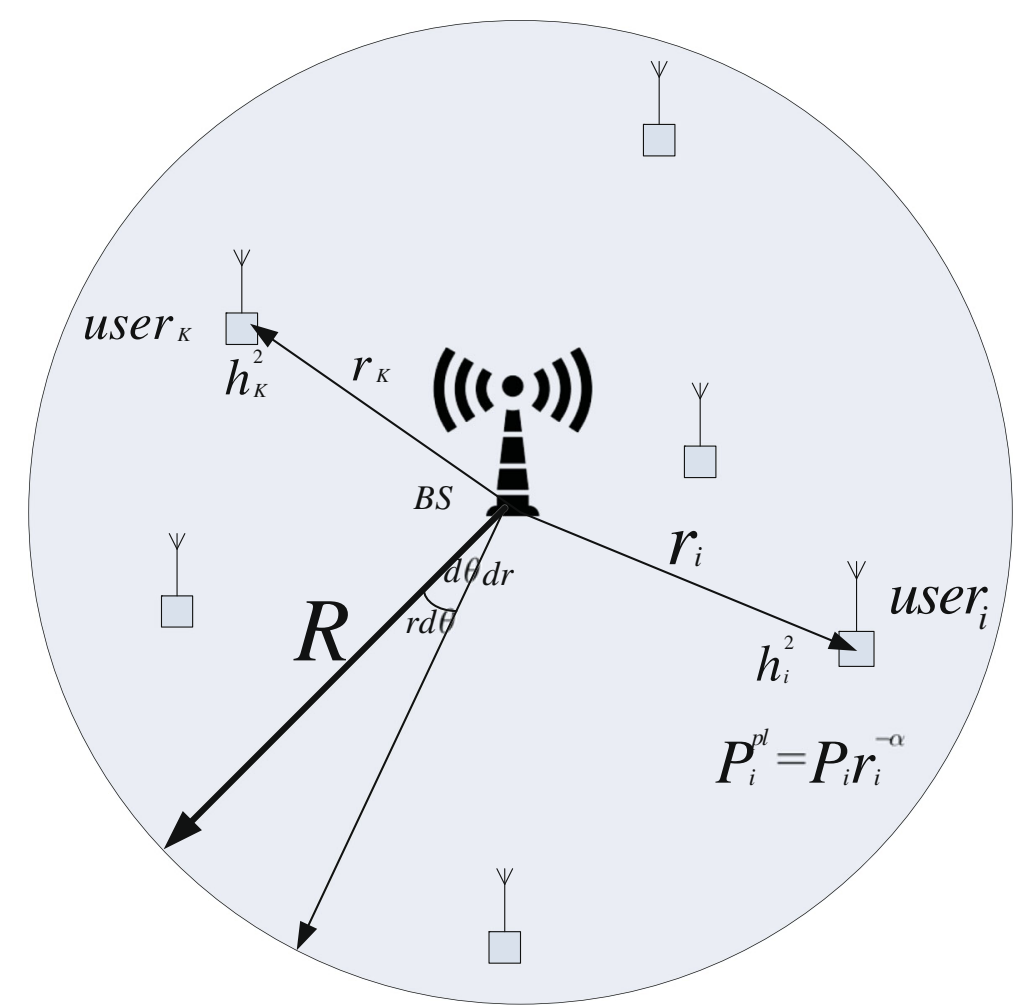

Fig. 2 Single-cell scenario with omni antenna at the BS. $R$ cell radius, $P_{i}$ received power, $h$ fading coefficient, $r$ user distance, and $K$ number of users 


$$
\begin{aligned}
\eta_{s}^{d}(R, \alpha)= & \frac{2}{\alpha \log 2} \sum_{i=1}^{K} \sum_{k=1}^{m} \sum_{l=1}^{m-k} \frac{(m-k) !}{l !} \\
& \times\left(\frac{\bar{\gamma}_{i}}{m}\right)^{k-m-l} R^{\alpha(m-k+l)}
\end{aligned}
$$

It is apparent that the downlink spectral efficiency is a function of the cell radius, path loss exponent, and signalto-noise ratio.

\subsubsection{Energy efficiency}

The energy efficiency function can be defined as $\eta(x) / x$, $x \in[0, X]$ and indicates the system's resource [24]. In the analysis of the cellular system, $\eta(x)$ represents the downlink data rate and $x$ denotes the signal-to-noise ratio, which depends on the transmitted power at the BS, noise and channel effects. For a single cell, the downlink energy efficiency can be shown to be given by:

$$
\eta_{E}^{d}=\sum_{i=1}^{K} \mathbb{E}\left[\frac{\log _{2}\left(1+\frac{P_{i}^{p l} h_{i}^{2}}{N_{o} B}\right)}{\frac{p_{i}^{p l} h_{i}^{2}}{N_{o} B}} \mid h_{i}^{2}\right],[\text { bits } / J]
$$

The expectation operation in (12) is done using the pdf of the channel [25] assuming that $h_{i}^{2}$ are independent random variables. Using [26, Eq. (8.4.6/5), Eq. (8.4.3/1), Eq. (2.24.3/1)], the downlink energy efficiency of a single-cell system over Nakagami- $m$ fading channel can be obtained in closed-form as a function of the downlink average SNR and fading scale parameter as

$$
\begin{aligned}
\eta_{E}^{d}\left(r_{i}, \alpha\right)= & \frac{(m)^{m}}{\Gamma(m) \log 2} \sum_{i=1}^{K} \frac{r_{i}^{\alpha(m+1)}}{\bar{\gamma}_{i}^{m+1}} \\
& \times G_{2,3}^{3,1}\left(\frac{m r_{i}^{\alpha}}{\bar{\gamma}_{i}} \mid \begin{array}{c}
-m, 1-m \\
0,-m,-m
\end{array}\right)
\end{aligned}
$$

where G[.] is the Meijer's G-function [26]. Since $r_{i}$ is a random variable, energy efficiency as a function of cell radius can be obtained by averaging (13) over the pdf of $r_{i}$. Using [23, Eq. (07.34.21.0003.01)], a closed-form can be obtained and is given by

$$
\begin{aligned}
\eta_{E}^{d}(R, \alpha)= & \frac{(m)^{m} R^{\alpha(m+1)-1}}{\alpha \Gamma(m) \log 2} \sum_{i=1}^{K} \frac{1}{\bar{\gamma}_{i}^{m+1}} \\
& \times G_{3,4}^{3,2}\left(\frac{m R^{\alpha}}{\bar{\gamma}_{i}} \mid \begin{array}{c}
-(m+1 / \alpha),-m,-m \\
0,-m,-m,-(m+1+1 / \alpha)
\end{array}\right)
\end{aligned}
$$

\subsection{Analysis of multi-cell scenarios}

In this section, the cellular network with regular hexagonal topology as shown in Fig. 1 is considered, in which co-channel interference is inevitable. The primary interest is focused on the analysis of downlink spectral and energy efficiencies by taking into account the interference and the channel effects.

\subsubsection{Signal-to-interference-plus-noise ratio model}

The instantaneous SINR given by (3) can be written as

$$
\bar{\gamma}_{j, i}^{I}=\frac{\frac{P_{j} \bar{h}_{j, i}}{N_{o} B} r_{j, i}^{-\alpha}}{1+\sum_{\substack{k=1 \\ k \neq j}}^{N_{I}} \frac{P_{k} \bar{h}^{2} k, i}{N_{o} B} D_{k, i}^{-\alpha}},
$$

where $\frac{P_{j, i} h_{j, i}^{2}}{N_{o} B}$ is the instantaneous SNR. Assuming all BSs transmit the same power and $h_{k, i}^{2}$ and $h_{j, i}^{2} \sim \mathcal{G}(m, \Omega)$, implies that $\bar{\gamma}_{j, i}=\frac{P_{j} \bar{h}_{j, i}}{N_{o} B}$ and $\bar{\gamma}_{k, i}=\frac{P_{k} \bar{h}_{k, i}}{N_{o} B}$. Also, it is noted that $\bar{\gamma}_{j, i} \propto r_{j, i}^{-\alpha}$ and $\bar{\gamma}_{k, i} \propto D_{k, i}^{-\alpha}$ and hence the average SINR can be written as [12]

$$
\bar{\gamma}_{j, i}^{I}=\frac{\bar{\gamma}_{j, i} r_{j, i}^{-\alpha}}{1+\Xi\left(N_{I}, D_{k}, r_{i}, \alpha, \bar{\gamma}_{k, i}\right)}
$$

where $\Xi=\sum_{\substack{k=1 \\ k \neq j}}^{N_{I}}\left(\frac{D_{k, i}}{r_{j, i}}\right)^{-\alpha} \bar{\gamma}_{k, i}$ is the interference term, which depends on network geometry. Since the investigation is based on the highest co-channel interference power, the user is located at the cell boundary, $r_{j, i}=R$. The distance $D_{k, i}$ for each co-channel interfere is determined in terms of $R$ and $D$. For three cases of BS antenna configurations, the average SINR is expressed as a function of normalized reuse distance, $R_{d}=D / R$. In the case of omni antenna configuration, the $i$ th user is located at the boundary of cell $\mathrm{BS}_{j=0}$. Thus, the other co-channel cells $\mathrm{BSs}_{j=1, . .6}$ are sharing the same frequency band and contribute to co-channel interference at the $i$ th user. The aggregated interference power, $\Xi$, is a function of normalized reuse distance and user location angle and path-loss exponent and is given by

$$
\begin{aligned}
\Xi^{o}\left(\theta, R_{d}, \alpha\right)= & \left(R_{d}-\sin \left(\theta-\frac{\pi}{3}\right)\right)^{-\alpha} \bar{\gamma}_{1, i}+\left(R_{d}-\sin \left(\theta+\frac{\pi}{3}\right)\right)^{-\alpha} \bar{\gamma}_{2, i} \\
& +\left(R_{d}+\sin \left(\theta-\frac{\pi}{3}\right)^{-\alpha} \bar{\gamma}_{3, i}+\left(R_{d}+\sin \left(\theta+\frac{\pi}{3}\right)^{-\alpha} \bar{\gamma}_{4, i}\right.\right. \\
& +\left(R_{d}+\sin (\theta)\right)^{-\alpha} \bar{\gamma}_{5, i} \\
& +\left(R_{d}-\sin (\theta)\right)^{-\alpha} \bar{\gamma}_{6, i}
\end{aligned}
$$

where $\theta$ is the user's angle, $0 \leq \theta \leq 2 \pi$. When sectorization concept is used in the system, the frequency bands in each cell are disjointly divided into sets and used in different sectors of the cell. Consequently, the co-channel interference is going to be less severe, which leads to better system efficiency. Cell sectorization can be used to improve cellular system capacity and has been investigated [21]. In this paper, two cell sectorization techniques are considered: three sectors in each cell with $120^{\circ}$ 
directivity antennas and six sectors in each cell with $60^{\circ}$ directivity antenna as shown in Fig. 3. In the case of $120^{\circ}$ antenna configuration, there are two $\mathrm{BS}_{j=1} \mathrm{BS}_{j=2}$ that create co-channel interference on the $i$ th user in $\mathrm{BS}_{j=0}$ as shown in Fig. 3a. The interference term can be expressed as function of $R_{d}, \alpha$ and $\theta$ as

$$
\begin{aligned}
\Xi^{120^{o}}\left(\theta, R_{d}, \alpha\right)= & \left(R_{d}+\sin \left(\theta+\frac{\pi}{6}\right)\right)^{-\alpha} \bar{\gamma}_{1, i} \\
& +\left(R_{d}+\sin \theta\right)^{-\alpha} \bar{\gamma}_{2, i}, 0<\theta<\frac{2 \pi}{3}
\end{aligned}
$$

Similarly, for the case of $60^{\circ}$ antenna configuration, it is noted that there is only one $\mathrm{BS}, \mathrm{BS}_{j=1}$, that creates cochannel interference on $i$ th user as shown in Fig. $3 \mathrm{~b}$ and the corresponding interference can be expressed as

$$
\Xi^{60^{\circ}}\left(\theta, R_{d}, \alpha\right)=\left(R_{d}+\sin \left(\theta+\frac{\pi}{4}\right)\right)^{-\alpha} \bar{\gamma}_{1, i}, 0<\theta<\frac{\pi}{3}
$$

Using the knowledge of average SINR for each antenna configuration, the spectral and energy efficiencies can be obtained using (10) and (13) where the users are randomly located at the cell boundary.

\subsection{Numerical results of SE and EE}

This section presents numerical results for SE and EE for single- and multi-cell networks. For the case of single-cell network, the SE and EE are evaluated using (11) and (14), and for multi-cell network, Monte Carlo simulation has been performed using (10) and (13). The steps used in simulation are given below, and the system parameters used are given in Table 1.
Table 1 Parameters used for simulation of SE, EE, SSE, and SEE

\begin{tabular}{lll}
\hline Symbol & Description & Value \\
\hline $\mathrm{R}$ & Cell radius & $1 \mathrm{~km}$ \\
$\alpha$ & Path loss exponent & 4 \\
$\bar{\gamma}$ & Signal-to-noise ratio (SNR) & $0-20 \mathrm{~dB}$ \\
$R_{d}$ & Normalized reuse distance & $2-6$ \\
$N_{l}$ & Number of interfering cells & 6 \\
$\theta$ & User's angle & $0-2 \pi, \frac{2 \pi}{3}, \frac{\pi}{3}$ \\
$m$ & Fading figure & $m \geq 1$ \\
\hline
\end{tabular}

1. The user's position is randomly selected using (5) and (6).

2. The distance of co-channel interference is obtained from Figs. 1 and 3 as a function of $R$ and $D$.

3. The interference $\Xi$ is calculated using (17), (18), and (19) for a given BS antenna configuration.

4. The user's SINR at the user's location, $\bar{\gamma}_{j, i}^{I}$, is determined using (15) and (16).

5. The SE and EE are calculated using (10) and (13).

For single- and multi-cell networks, the SE and EE are plotted as a function of SNR for omni, $120^{\circ}$, and $60^{\circ}$ sectorized antenna systems over Nakagami- $m$ fading channel in Figs. 4 and 5, respectively. It is observed that SE is an increasing function of SNR whereas EE decreases monotonically as SNR increases. The single-cell network is superior in terms of SE and EE compared to multi-cell networks as single-cell networks are devoid of co-channel interference. However, for sectorized antenna systems, it is possible to obtain improved SE and EE metrics. For example, the SE and EE for the $60^{\circ}$ system approaches that of a single-cell system and these metrics for $120^{\circ}$ system

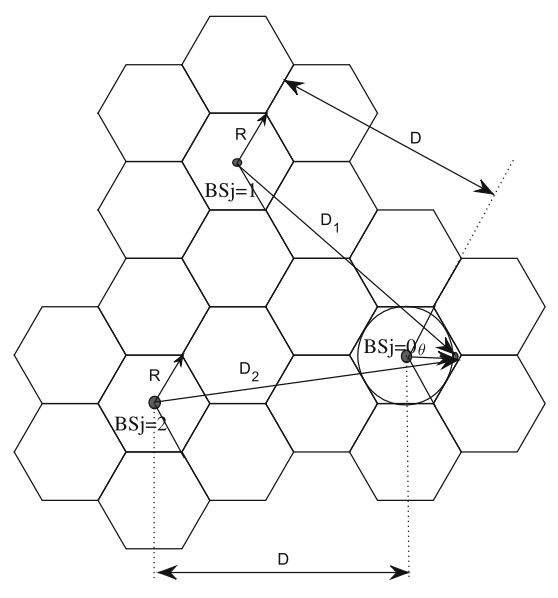

(a)

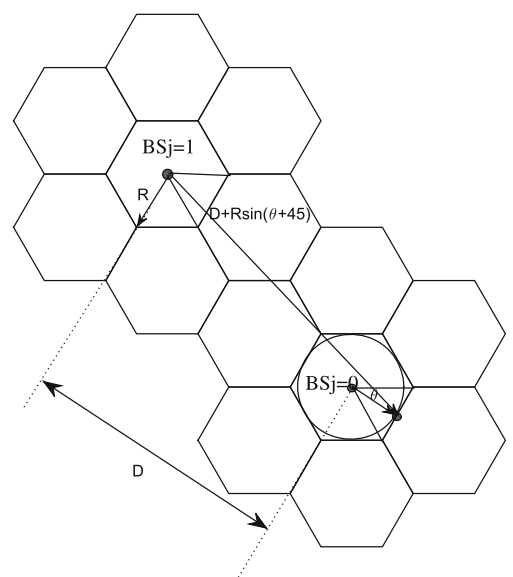

(b)

Fig. 3 Hexagonal cellular network with $120^{\circ}$ and $60^{\circ}$ sectorized antennas configurations. $R$ cell radius, $D$ reuse distance, and $D_{i}$ interfering distance. a $120^{\circ}$ directivity. $\mathbf{b} 60^{\circ}$ directivity 


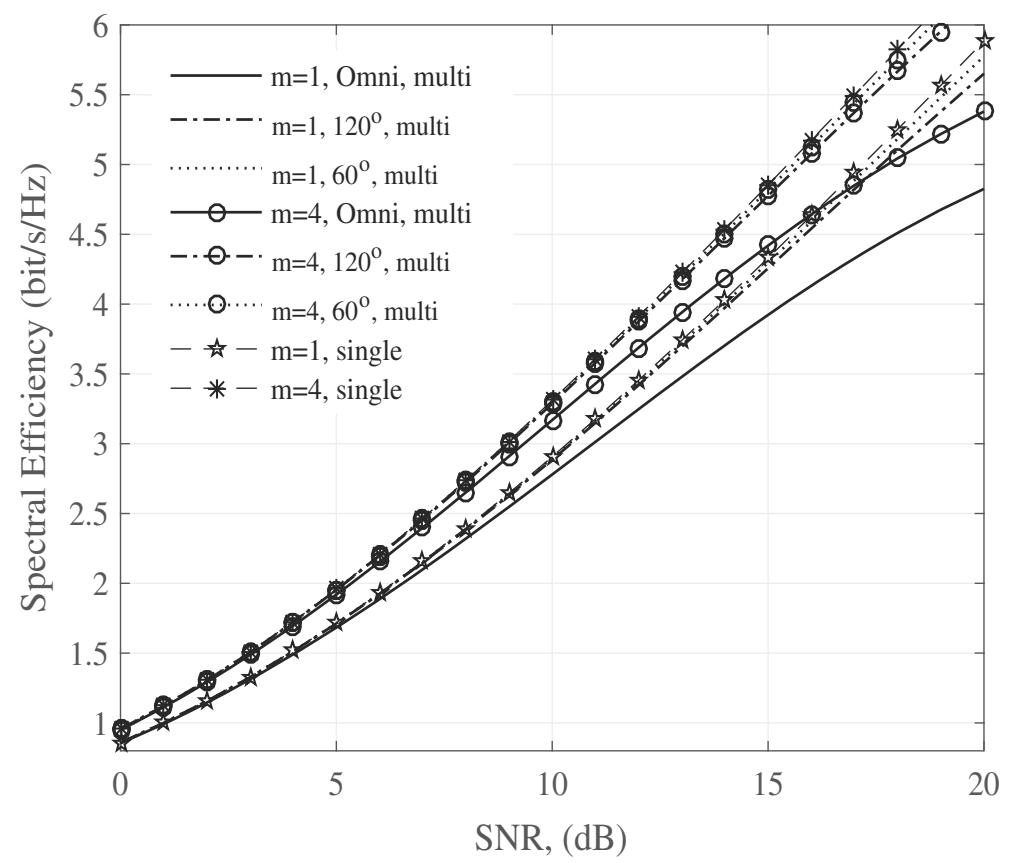

Fig. 4 Spectral efficiency in (bit/s/Hz) for single- and multi-cell cellular networks for three different antenna configurations as a function of SNR for various values of fading figure, $m$, with $R_{d}=3.5$ and $\alpha=4$. SE of multi-cell network with omni antenna system for $m=1$ and 4 , SE of multi-cell network with $120^{\circ}$ sectorized antenna system for $m=1$ and 4 , SE of multi-cell network with $60^{\circ}$ sectorized antenna system form $=1$ and 4 , SE of single-cell network for $m=1$ and 4

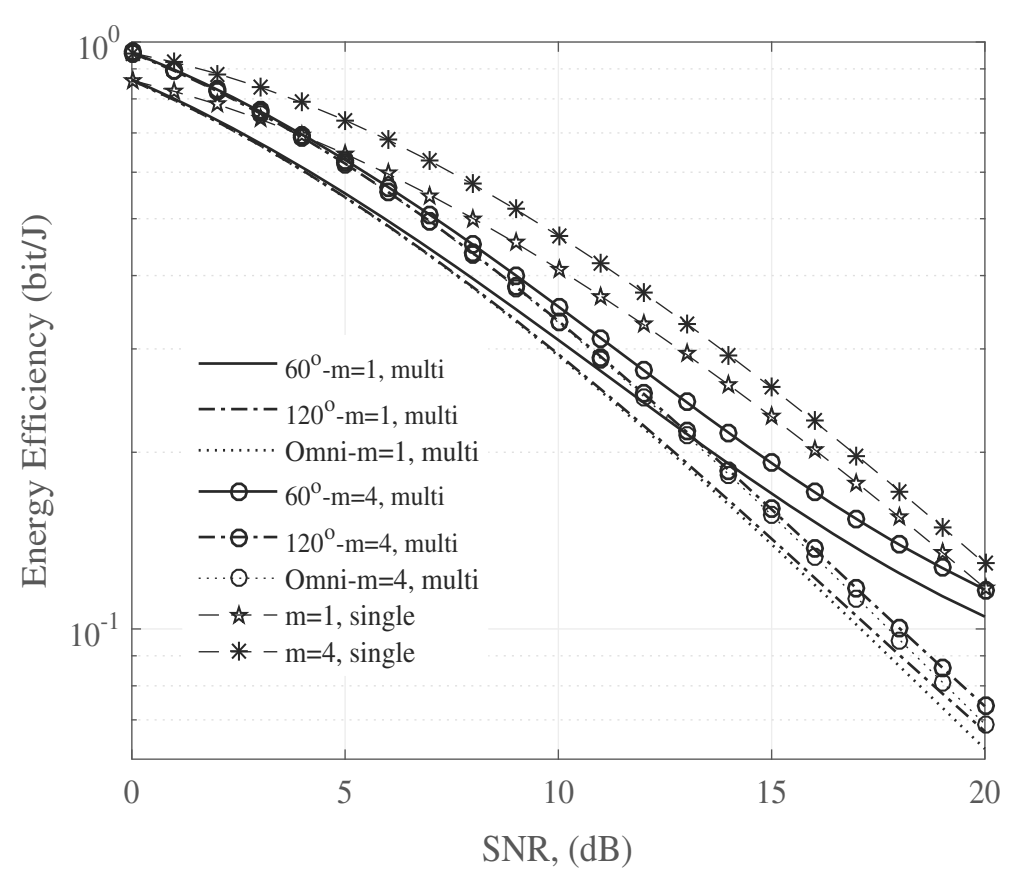

Fig. 5 Energy efficiency in (bit/J) for single- and multi-cell cellular networks for three different antenna configurations as a function of SNR for various values of fading figure, $m$, with $R_{d}=3.5$ and $\alpha=4$. EE of multi-cell network with omni antenna system for $m=1$ and 4 , EE of multi-cell network with $120^{\circ}$ sectorized antenna system for $m=1$ and 4 , EE of multi-cell network with $60^{\circ}$ sectorized antenna system for $m=1$ and 4 , EE of single-cell network for $m=1$ and 4 
is far better than the omni system at high SNR. In contrast, the overall SE of multi-cell system is much higher than a single-cell system. As given by (17)-(19), the interference $\Xi$ is a function of $R_{d}$, and therefore, SE and EE are function of $R_{d}$ for fixed SNR and the antenna configuration. Figure 6 illustrates the SE for multi-cell systems for Nakagami- $m$ channel and these antenna configurations as a function of $R_{d}$. It is observed that SE improves as $R_{d}$ increases, which is due to low co-channel interference in the system. As can be seen, $60^{\circ}$ system achieves better SE than the $120^{\circ}$ and the omni systems. Also, it is evident that fading channel impacts the SE particularly at high $R_{d}$ for the omni system. The EE is plotted as a function of $R_{d}$ in Fig. 7. It is obvious that EE, for all these antenna configurations, decreases as $R_{d}$ is increased, which implies more transmission power is required at the BS. It is also noted that EE for each antenna system approaches the same value as $R_{d}$ is increased indicating less co-channel interference at the user.

\section{Affected area, spatial SE, and spatial EE}

In the previous section, cellular networks were analyzed using SE and EE without considering the spatial characteristics of the cellular system. Expanding the coverage area is an important issue in design of cellular networks, as an increase of the reuse distance $D$ expands the coverage area in the network. The given power transmitted by the BS can affect the QoS of the network for large value $D$, and hence, $\mathrm{SE}$ and $\mathrm{EE}$ are required to be examined as a function of coverage area. The spatial SE and EE metrics have been used in $[15,16]$ to examine a cellular system. The spatial SE (SSE) quantifies how many $\mathrm{bit} / \mathrm{s} / \mathrm{Hz} / \mathrm{unit}$ of area of the network can be delivered to the user, and it characterizes the spectral utilization of the cellular system for a certain coverage area. Also, spatial EE (SEE) is defined as the maximum energy efficiency of a cellular network divided by the coverage area. These definitions capture the trade-offs between SSE and SEE of the network. These spatial efficiency metrics depend on the SE and EE of cellular system introduced in Section 3 and the area covered by a BS transmission signal.

\subsection{Affected area}

For the single-cell scenario shown in Fig. 2, the affected area is the area where a considerable amount of BS transmission power is detected above a certain threshold [18]. This area is a function of BS transmission power, propagation environment, and BS antenna configuration. Hence, the affected area is a useful metric that can be used to quantify the spatial properties of a cellular system. Assuming the BS coverage to be circular as shown in Fig. 2, the affected area can be written as

$$
\mathcal{A}_{a}=\int_{0}^{2 \pi} \int_{0}^{\infty} F_{a}\left(\gamma_{t}, r\right) r d r d \theta
$$

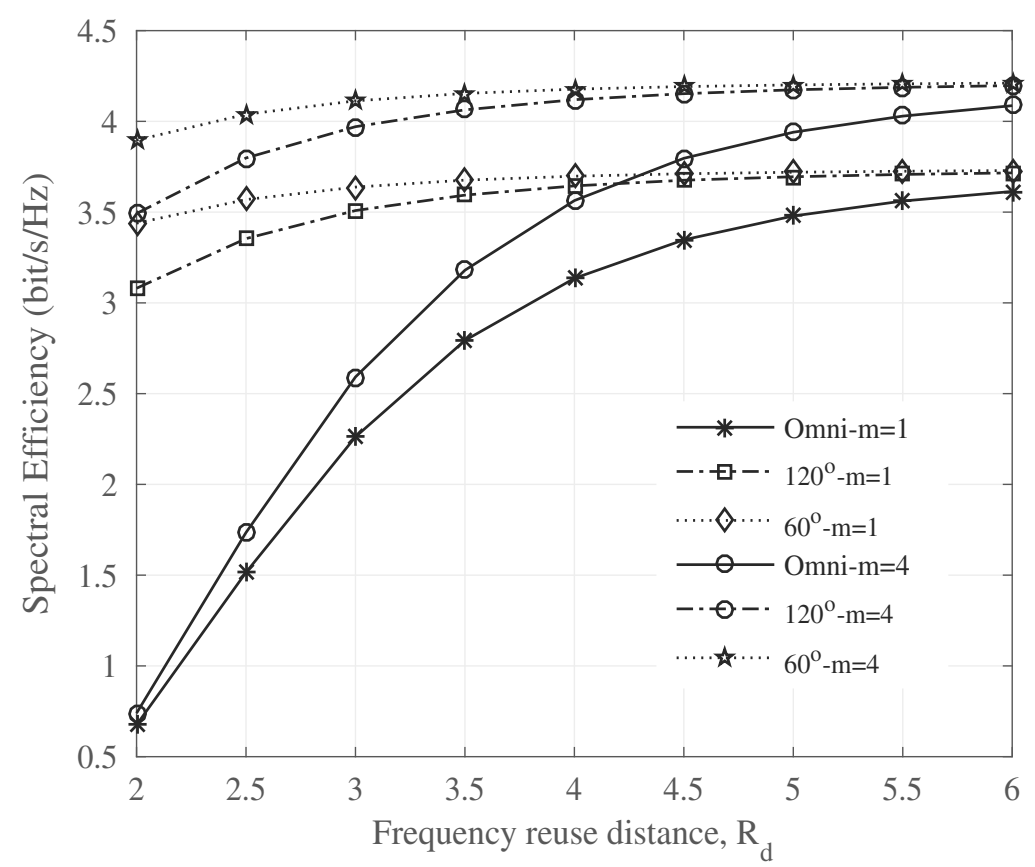

Fig. 6 Spectral efficiency of cellular network for omni, $120^{\circ}$ and $60^{\circ}$ antenna configurations as a function of normalized reuse distance with fading figure, $m=1$ and $4, \mathrm{SNR}=10 \mathrm{~dB}$, and $\alpha=4$. SE of multi-cell network with omni antenna system for $m=1$ and 4 SE of multi-cell network with $120^{\circ}$ sectorized antenna system form $=1$ and 4 , SE of multi-cell network with $60^{\circ}$ sectorized antenna system for $m=1$ and 4 


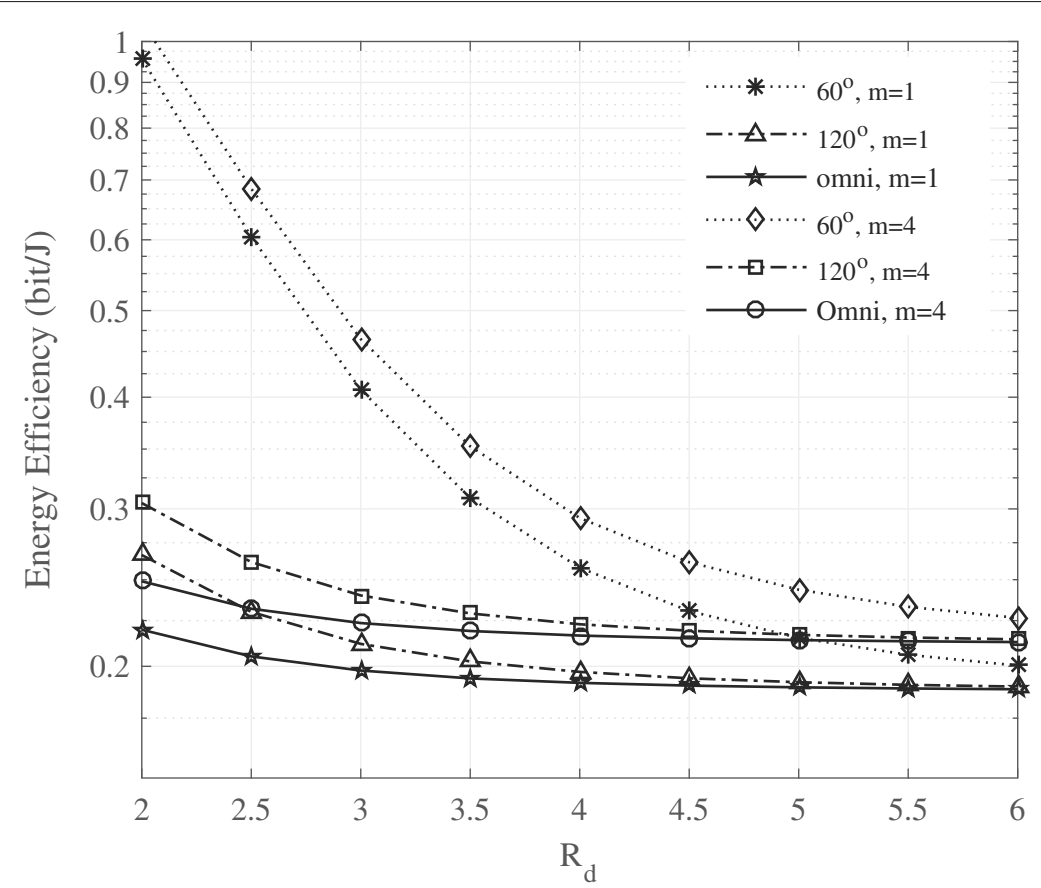

Fig. 7 Energy efficiency of cellular network for omni, $120^{\circ}$ and $60^{\circ}$ antenna configurations as a function of normalized reuse distance with fading figure, $m=1$ and $4, \mathrm{SNR}=10 \mathrm{~dB}$, and $\alpha=4$. EE of multi-cell network with omni antenna system for $m=1$ and 4 , EE of multi-cell network with $120^{\circ}$ sectorized antenna system for $m=1$ and 4 , EE of multi-cell network with $60^{\circ}$ sectorized antenna system for $m=1$ and 4

where $F_{a}\left(\gamma_{t}, r\right)$ is the affected probability. $F_{a}(.,$.$) denotes$ the probability that $\gamma_{i}$ is greater than specified SNR threshold $\gamma_{t}$ at a user's distance $r$ and is

$$
F_{a}\left(\gamma_{t}, r\right)=\mathbb{P}\left[\gamma>\gamma_{t}\right]
$$

where $\gamma$ has pdf $f_{\gamma}(\gamma)$ and it is given by (7). For Nakagami$m$ channel, $F_{a}$ is obtained as

$$
F_{a}\left(\gamma_{t}, r\right)=\frac{\Gamma\left(m, m \frac{\gamma_{t}}{\gamma} r^{\alpha}\right)}{\Gamma(m)}
$$

By substituting (22) into (20), the affected area for Nakagami- $m$ channel can be derived with the aid of ([26], Eq. (8.4.16/2), Eq. (2.24.2/1)) as

$$
\mathcal{A}_{a}^{N}=\frac{2 \pi}{\alpha}\left(m \frac{\gamma_{t}}{\bar{\gamma}}\right)^{-2 / \alpha} \frac{\Gamma\left(\frac{2}{\alpha}\right) \Gamma\left(\frac{2}{\alpha}+m\right)}{\Gamma\left(\frac{2}{\alpha}+1\right) \Gamma(m)}
$$

The affected area is plotted as a function of average SNR for specific parameters $\gamma_{t}=10$ and $15 \mathrm{~dB}, \alpha=4$, and $m=1,4$ in Fig. 8 . It is observed that when $m=1$ less area is covered, which indicates the fading channel impacts the affected area. Also, it is noted that low threshold level provides large affected area.

\subsection{Spatial spectral and energy efficiencies for single-cell scenario}

For a single cell, the SSE can be defined as the ratio of the average $\mathrm{SE}$ of a cell over the affected area of the BS transmission as

$$
\operatorname{SSE}_{S c}^{d}=\frac{\eta_{s}^{d}(R, \alpha)}{\mathcal{A}_{a}\left(\gamma_{t}, \alpha\right)}
$$

where $\eta_{s}^{d}(R, \alpha)$ and $\mathcal{A}_{a}\left(\gamma_{t}, \alpha\right)$ are given by (11) and (23), respectively. Using the same concept, the SEE can be obtained as

$$
S E E_{S c}^{d}=\frac{\eta_{E}^{d}(R, \alpha)}{\mathcal{A}_{a}\left(\gamma_{t}, \alpha\right)},
$$

where $\eta_{E}^{d}(R, \alpha)$ is given by (14). The SSE and SEE are plotted as a function of SNR for different channel parameters and the SNR threshold, and $\alpha=4$ in Figs. 9 and 10, respectively. Figure 9 demonstrates that SSE attains the maximum values in a certain region of SNR, which is the optimum range of operating SNR. Also, it is evident that the affected area has considerable effect on the overall SSE specifically at high values of $\mathcal{A}_{a}^{N}$, as illustrated in Fig. 8. For example for $\gamma_{t}=10 \mathrm{~dB}$ and $m=4$, SSE is below $1.2 \mathrm{bit} / \mathrm{s} / \mathrm{Hz} \mathrm{km}^{2}$ and refers to a large covered area. 


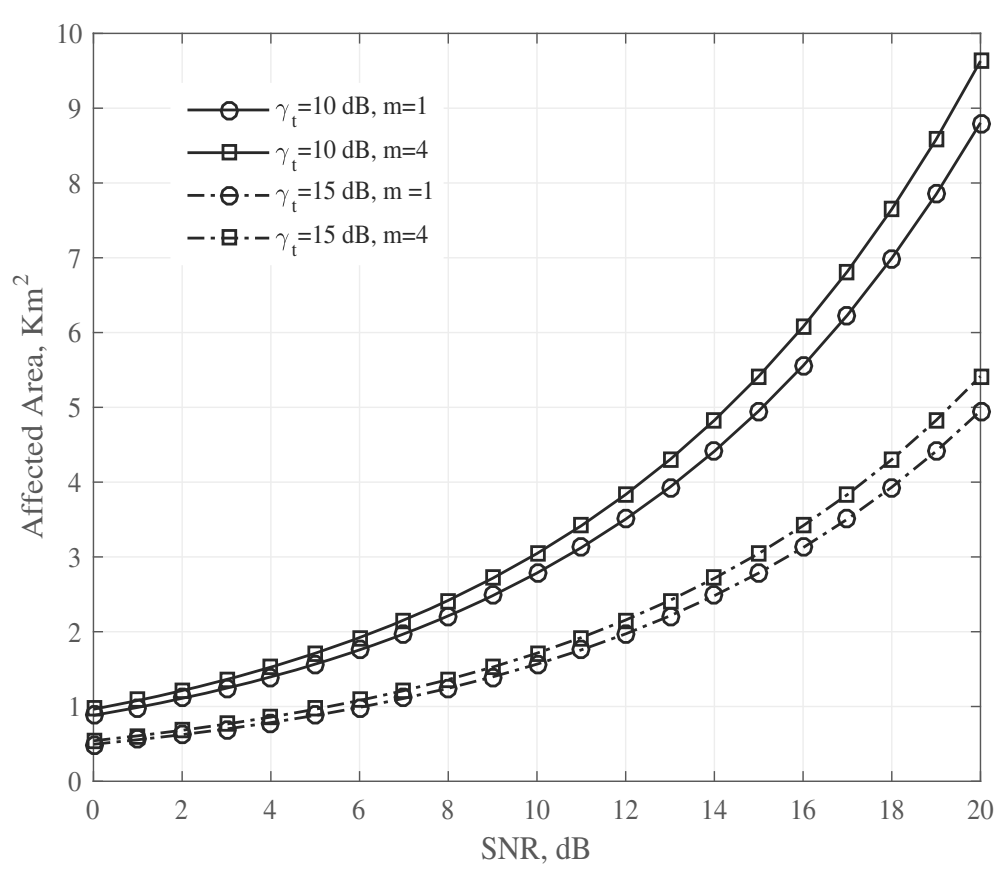

Fig. 8 Affected area as a function of SNR for fading figure, $m=1$ and 4, SNR threshold $\gamma_{t}=10$ and $15 \mathrm{~dB}$, and path loss exponent $\alpha=4$. Affected area for, $m=1$ and 4 , and $\gamma_{t}=10$ and $15 \mathrm{~dB}$

From Fig. 10, it can be seen that SEE is an exponentially decreasing function of SNR, and the high SNR threshold provides better SEE. It is observed from Figs. 8 and 9, the optimum range of SNR is in the range $2 \leq \bar{\gamma} \leq$ $10 \mathrm{~dB}$, which provides the highest values of SSE and acceptable SEE.

\subsection{Spatial spectral and energy efficiencies for multi-cell scenario}

For multi-cellular networks shown in Figs. 1 and 3, the SSE and SEE can be defined as the ratio of the average SE and $E E$ of the cellular system to the covered area. In this paper, the frequency reuse distance $D$ is considered and

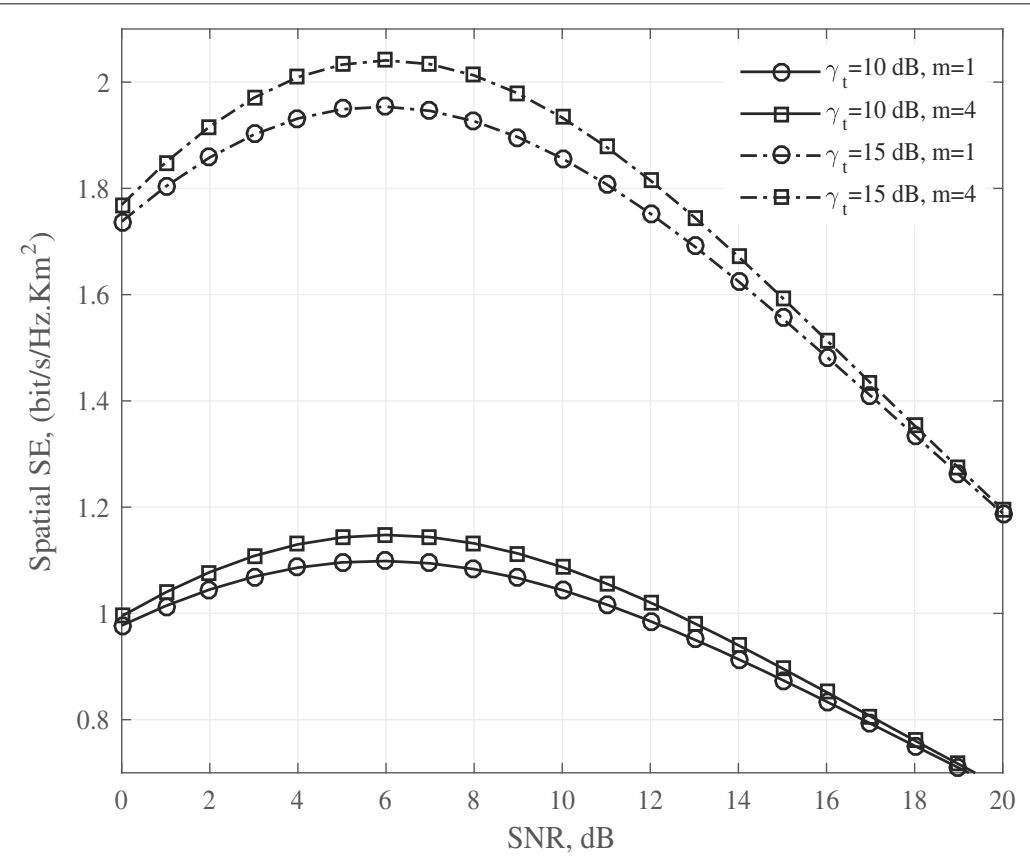

Fig. 9 Spatial spectral efficiency in (bit/s/Hz km²) for single cell as a function of SNR for fading figure $m=1$ and 4 , SNR threshold $\gamma_{t}=10$ and 15 , and path loss exponent $\alpha=4$. Spatial SE for, $m=1$ and 4 , and $\gamma_{t}=10$ and 15 


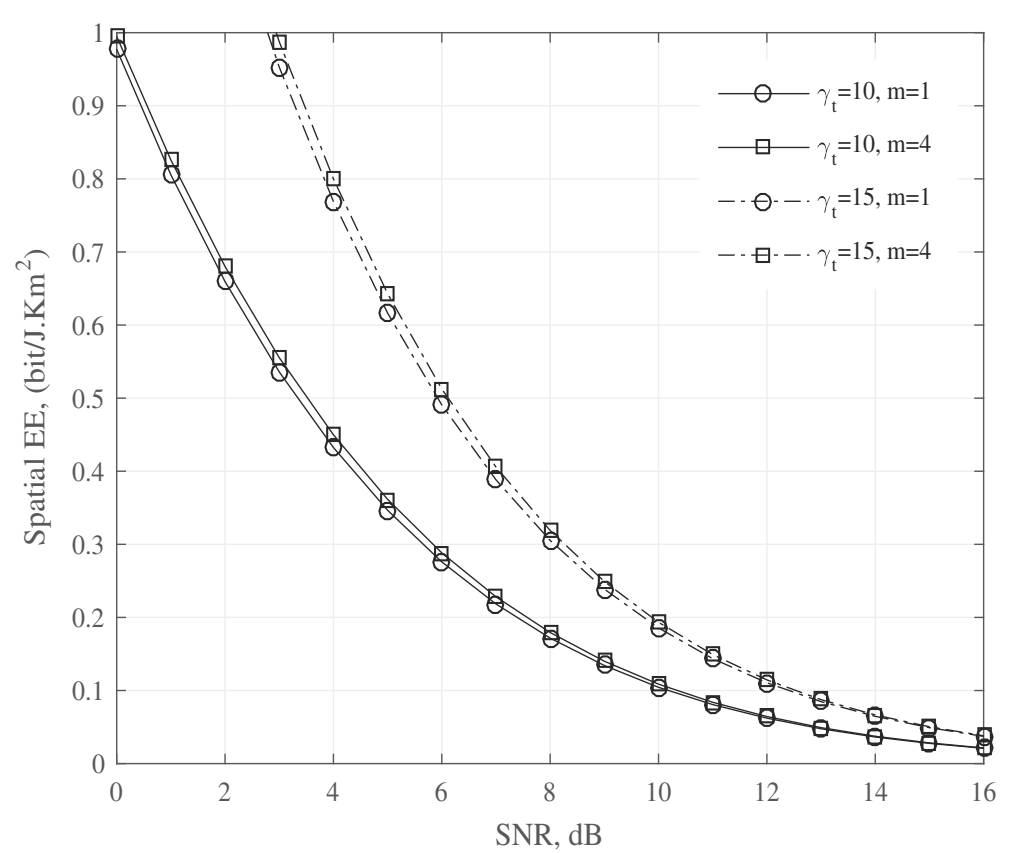

Fig. 10 Spatial energy efficiency in (bit/J.Km²) for single cell as a function of SNR for fading figure $m=1$ and 4 , SNR threshold $\gamma_{t}=10$ and 15 , and path loss exponent $\alpha=4$. Spatial EE for, $m=1$ and 4 , and $\gamma_{t}=10$ and $15 \mathrm{~dB}$

then the corresponding covered area is $\pi D^{2} / 4 n$, where $n$ is the sectorization factor. The value of $n=1,3$ and 6 for omni, $120^{\circ}$ and $60^{\circ}$, respectively. The SSE and SEE can be formulated as [15]

$$
\begin{aligned}
& S S E_{m c}^{d}=4 n \frac{\eta_{s}^{d}\left(r_{i}, \alpha\right)}{\pi R_{d}^{2} R^{2}}, \\
& S E E_{m c}^{d}=4 n \frac{\eta_{E}^{d}\left(r_{i}, \alpha\right)}{\pi R_{d}^{2} R^{2}},
\end{aligned}
$$

Since the SSE and SEE are functions of $m$, SNR, and $R_{d}$, they are plotted as functions of SNR and $R_{d}$ for $m=4$ using simulation of omni, $120^{\circ}$ and $60^{\circ}$ directivity antenna multi-cellular systems using the parameters given in Table 1. Figure 11 depicts the SSE for multi-cell scenario for three antenna configurations as a function of SNR and $R_{d}$. It is observed that SSE of the $60^{\circ}$ system is superior to omni and $120^{\circ}$ systems as co-channel interference is minimum in the $60^{\circ}$ system. Additionally, SEE is plotted as a function of SNR and $R_{d}$ in Fig. 12. As can be seen from the figure, SEE degrades as $R_{d}$ increases. It is noted that as $R_{d}$ increases, the coverage area also increases, and to maintain the same SSE, the BS is required to pump more power. Furthermore, it is observed that while SSE is an increasing function of SNR, SEE is a decreasing function of SNR. From Figs. 11 and 12, we find that the sectorized antenna system can be gainfully employed for designing an energyefficient multi-cellular network; however, antenna system complexity is increased. Shrinking the reuse distance in the network implies reducing the coverage area which will increase the SSE. However, the co-channel interference will increase which will decrease SSE. Thus, it is clear from Fig. 11 that there a is trade-off between these two metrics that ensure SSE stability for $R_{d}$ above 4 . Figure 12 quantifies the fact that SEE progressively decreases with the reuse distance and SINR. From this observation, it can be concluded that the reuse distance of 4 has a negative effect on SEE and provides no improvement in SSE. The sectorization antenna system has a significant effect on the spatial efficiency metrics, which attain better SSE and SEE than for the omni system. This is due to the following: (i) the transmission power is oriented to a specific region, (ii) the co-channel interference is less, and (iii) the utilized area is reduced by factor $n$; however, the system is much more complex. As can be seen from these results, the efficiency metrics are always in conflict with SNR and $R_{d}$; hence, the trade-off between these parameters should be considered when designing multi-cell networks.

\section{Coverage probability in Nakagami- $m$ fading with co-channel interference}

Cellular network becomes more energy efficient when the transmitted power at the BS is reduced. However, the BS coverage cannot be guaranteed beyond a certain level of transmitted power. To precisely address the cellular network energy consumption issue, the coverage 


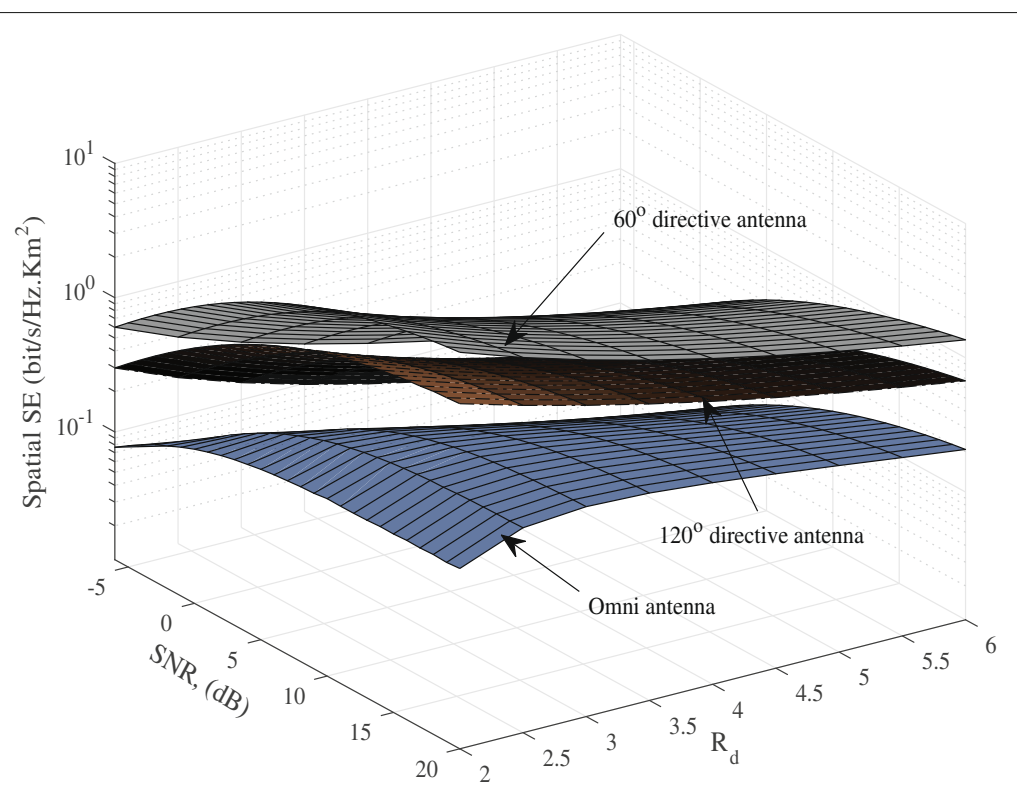

Fig. 11 Spatial spectral efficiency in (bit/s/ $\mathrm{Hz} \mathrm{km}^{2}$ ) of multi-cell cellular network as a function of SNR and normalized reuse distance, $R_{d}$ for omni, $120^{\circ}$ and $60^{\circ}$ antenna configurations with $m=4$ and $\alpha=4$. Spatial SE for omni antenna system, spatial SE for $120^{\circ}$ sectorized antenna system, spatial SE for $60^{\circ}$ sectorized antenna system

performance has to be studied in addition to the efficiency metrics. An efficient cellular deployment strategy that minimizes energy consumption has been investigated using coverage probability in [27]. The coverage probability of a user in the $j$ th cell is the probability that the SINR is greater than a certain threshold $\gamma_{t}$. For a reference user in the cellular network, it is defined as

$$
P_{c o v} \triangleq \mathbb{P}\left[\operatorname{SINR} \geq \gamma_{t}\right]
$$

Using (15), the coverage probability can therefore be written as

$$
P_{c o v} \triangleq \mathbb{P}\left[\gamma \geq \frac{\left(1 / \bar{\gamma}+\Xi\left(\alpha, r, N_{I}\right)\right) \gamma_{t}}{r^{-\alpha}}\right]
$$

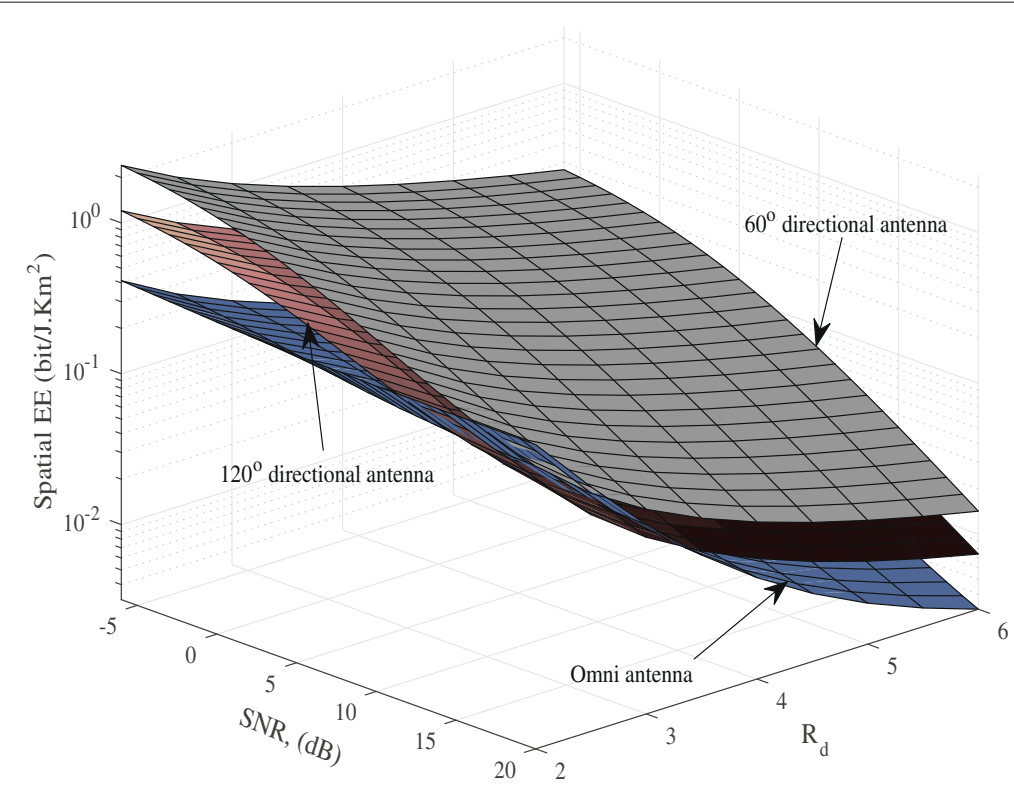

Fig. 12 Spatial energy efficiency in (bit/J $\mathrm{km}^{2}$ ) of multi-cell cellular network as a function of SNR and normalized reuse distance, $R_{d}$ for omni, $120^{\circ}$ and $60^{\circ}$ antenna configurations with $m=4$ and $\alpha=4$. Spatial EE for omni antenna system, spatial EE for $120^{\circ}$ sectorized antenna system, and spatial EE for $60^{\circ}$ sectorized antenna system 
where $\gamma \sim \mathcal{G}(m, \Omega)$ as described in (7). For a user in the Nakagami- $m$ fading environment, the $P_{c o v}$ can be obtained as [28]

$$
\begin{aligned}
& P_{c o v}=\left.\mathbb{E}\right|_{I, r}\left[F_{\gamma}\left(\frac{\left(1 / \bar{\gamma}+\Xi\left(\alpha, r, N_{I}\right)\right) \gamma_{t}}{r^{-\alpha}}\right)\right] \\
& P_{c o v}=\left.\frac{1}{\Gamma(m)} \mathbb{E}\right|_{I, r}\left[\Gamma\left(m, \frac{m\left(1+\Xi\left(\alpha, r, N_{I}\right)\right) \gamma_{t} \bar{\gamma}}{\bar{\gamma}^{2} r^{-\alpha}}\right)\right]
\end{aligned}
$$

The expectation is taken over the user's position as is modeled in (4), and the co-channel interference. Extensive simulations were conducted using the parameters shown in Table 1 to evaluate the coverage probability for three BS antenna scenarios. Without any loss of generality, the mobile user is assumed to be located at the cell boundary which is the worst case scenario. The variation of coverage probability with respect to SINR threshold is plotted in Fig. 13 for the three antenna configurations. Also, the impact of fading is illustrated using $P_{c o v}$ evaluated for several values of $m$. Figure 13 shows that the $60^{\circ}$ sectorization antenna achieves better coverage than the $120^{\circ}$ and omni antenna systems. On the other hand, high coverage probability is attained for low threshold values whereas poor coverage is expected for high thresholds. Also, it is obvious that more coverage is acquired for large values of $m$. Further coverage probability investigations have been done in terms of reuse frequency distance and path loss exponent. Figure 14 depicts how the coverage improves as the normalized reuse distance increases, which indicates lesser influence of co-channel interference. As can be seen, the coverage probability is highly impacted for small values of $R_{d}$ for the omni antenna system; in contrast, the coverage is still high with sectorization of antennas because the signal power is directed to a certain region and hence less interference is taking place. For large values of $R_{d}$, the coverage for the different antenna systems and path loss becomes tight and approaches unity. Also, it is observed that the propagation environment represented by $\alpha$, has a significant effect on the coverage probability. From the results obtained, it is evident that the coverage probability is consistent with SE and SSE as a function of SNR and $R_{d}$, which is clear in Figs. 4, 6 and 14, and supports the linking between these performance indexes [19]. In contrast, the coverage probability is incompatible with the EE and SEE as it is obvious in Figs. 7, 12 and 14. This implies that designing an energy efficient cellular network requires a trade-off between these efficiency metrics. In Table 2 a comparison of various metrics of a cellular network is provided.

The metrics in Table 2 that shows the superiority of sectorized antenna techniques over omni antenna system in the network. It is clear that the efficiency metrics and the coverage probability are boosted. For instance, the $60^{\circ}$ configuration enhances SE and EE by 58 and 107\%, respectively. Also, the spatial efficiencies have surged by

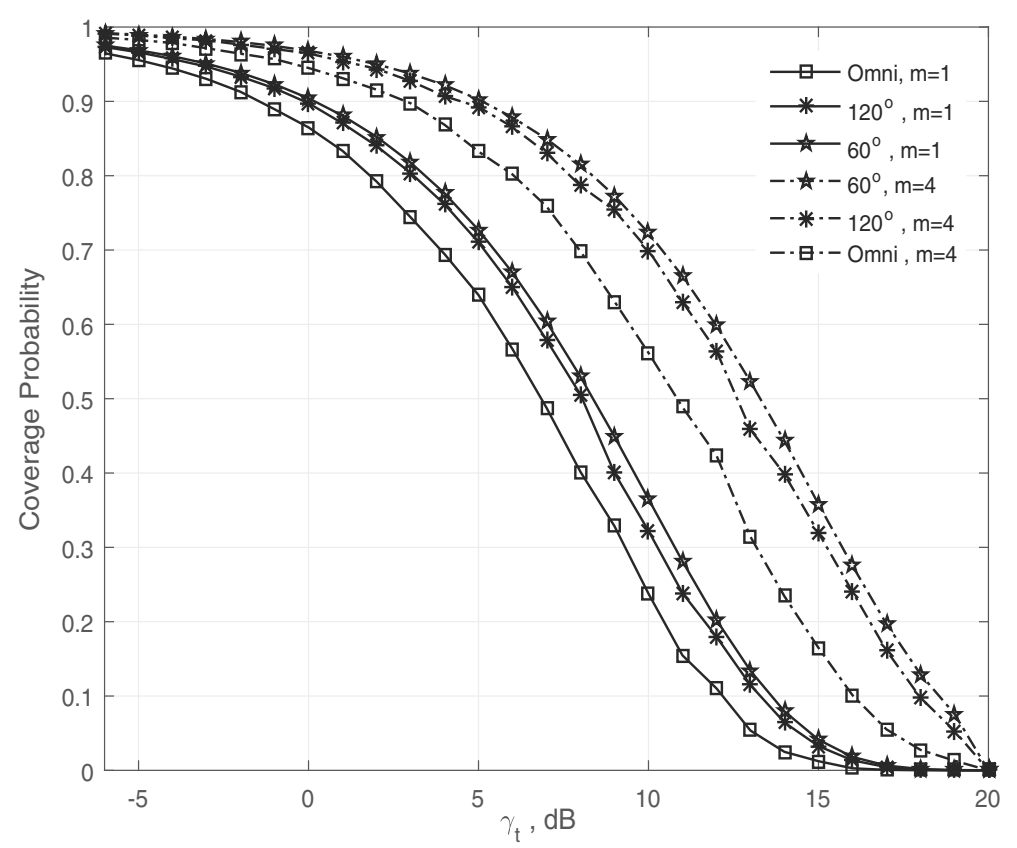

Fig. 13 Coverage probability of cellular network as a function of SNR threshold, $\gamma_{t}$, with $m=1$ and $4, \bar{\gamma}=10 \mathrm{~dB}, R_{d}=4$ and $\alpha=4$ for omni, $120^{\circ}$ and $60^{\circ}$ antenna configurations. Coverage probability for omni antenna with $m=1$ and 4 , Coverage probability $120^{\circ}$ sectorized antenna with $m=1$ and 4 , Coverage probability $60^{\circ}$ sectorized antenna with $m=1$ and 4 


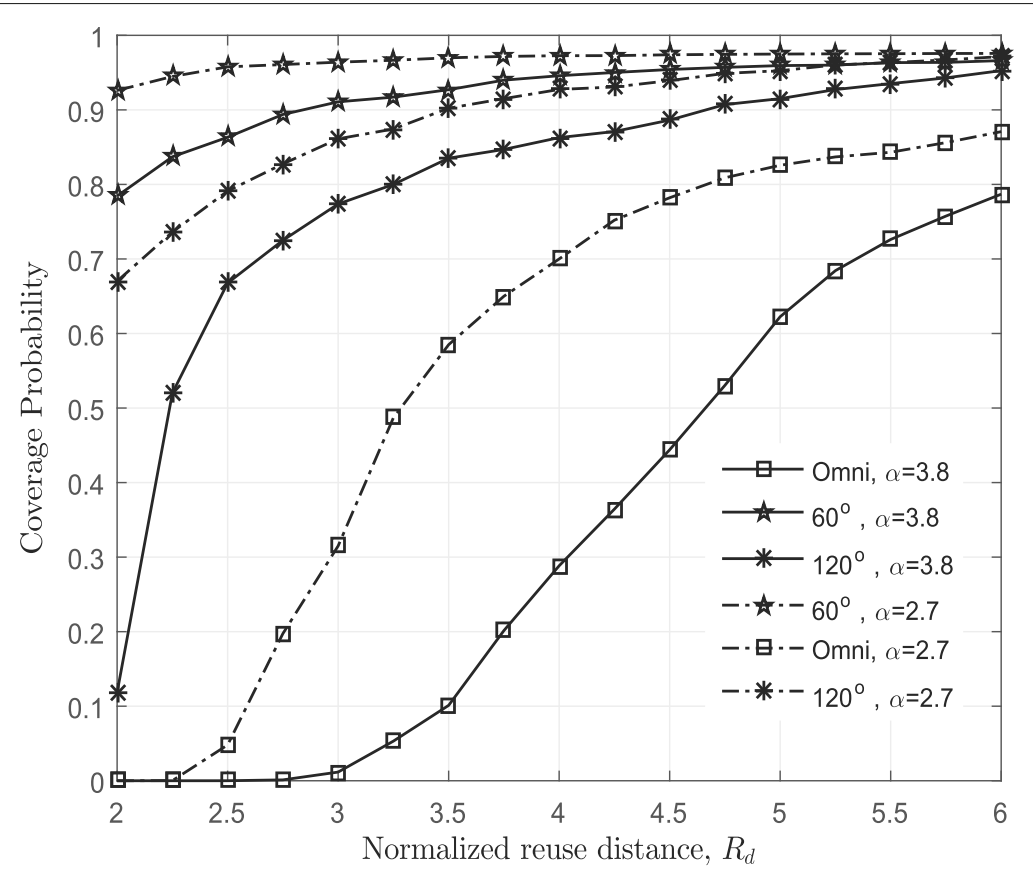

Fig. 14 Coverage probability of cellular network as a function of normalized reuse distance $R_{d}$ for omni, $120^{\circ}$ and $60^{\circ}$ antenna configurations with $m=3$, path loss exponent $\alpha=3.8$ and 2.7. Coverage probability for omni antenna with $\alpha=3.8$ and 2.7. Coverage probability $120^{\circ}$ sectorized antenna with $\alpha=3.8$ and 2.7. Coverage probability $60^{\circ}$ sectorized antenna with $\alpha=3.8$ and 2.7

about three times. The coverage probability approaches a value of unity, when sectorization technique is implemented in the cellular network.

\section{Conclusions}

This paper has presented a framework for designing green cellular networks using spatial spectral and energy efficiency metrics and coverage probability. Both theoretical derivations and simulations of these metrics for single- and multi-cell systems with omni and sectorized antenna systems in the cellular network were presented. The investigation in this paper has considered the effect of co-channel interference, signal propagation path loss, and multi-path fading channel on the efficiency metrics. The efficiency metrics have been derived and analyzed in terms of signal-to-interference-plus-noise ratio, reuse frequency distance, cell radius, path loss exponent, users'

Table 2 Comparison results for fixed values of $S N R=10 \mathrm{db}$, $R_{d}=3, \alpha=4$ and $m=4$

\begin{tabular}{llll}
\hline Metric & Omni & $120^{\circ}$ & $60^{\circ}$ \\
\hline Spectral efficiency bit/s/Hz & 2.6 & 3.63 & 4.11 \\
Energy efficiency bit/J & 0.22 & 0.24 & 0.46 \\
Spatial spectral efficiency bit/s/Hz/ $/ \mathrm{Km}^{2}$ & 0.32 & 1.35 & 2.76 \\
Spatial energy efficiency bit $/ \mathrm{J} / \mathrm{Km}^{2}$ & 0.074 & 0.15 & 0.29 \\
Coverage probability $\mid \gamma_{t}=10 \mathrm{~dB}$ & 0.420 & 0.862 & 0.945 \\
\hline
\end{tabular}

location, and fading scale. The results show that for design of efficient cellular network, there exist trade-offs among coverage probability, spatial energy efficiency, and spatial spectral efficiency which must be carefully considered in system implementation. The BS antenna sectorization technique can effectively be used to improve the overall energy efficiency of the system efficiencies but at the cost of increasing the complexity of the system.

\section{Methods}

The aim of this study is to present a framework for designing an energy-efficient cellular network. The network is assumed to consist of hexagonal cells with radius $R$ and reuse distance of $D$. Three BS antenna configurations namely omni, $120^{\circ}$, and $60^{\circ}$ are considered in the design. The design framework is based on spatial spectral and energy efficiencies and coverage probability metrics subject to co-channel interference, path loss, and Nakagami- $m$ propagation environment. The signalto-interference-plus-noise ratio (SINR) for an arbitrary user for the worst case location in a cell is first obtained. Next, the spatial spectral and energy efficiency and coverage probability metrics are described in terms of SINR. The SINR is a function of random location of the user, cell radius $R$, reuse distance $D$, path loss exponent $\alpha$, and statical parameters of propagation environment. Statistical averaging and Monte Carlo simulation are then used to evaluate the metrics. The process is carried out for each 
of three BS antenna configurations. Based on the numerical values of the metrics, energy efficient-cellular network designs are arrived at.

\section{Abbreviations}

AWGN: Additive White Gaussian Noise; BS: Base station; EARTH: Energy aware radio and network technologies; ECONET: Low-energy consumption networks; EE: Energy efficiency; pdf: Probability density function; QoS: Quality of service; SE: Spectral efficiency; SEE: Spatial energy efficiency; SINR:

Signal-to-interference-plus-noise ratio; SNR: Signal-to-noise ratio; SSE: Spatial spectral efficiency

\section{Acknowledgements}

The first author would like to thank the University of Tripoli, Libya, and the Libyan Ministry of Higher Education for their support and scholarship.

\section{Availability of data and materials}

The authors declare that the data of this research included the data used for simulation and generated during the study are available in a public repository.

\section{Authors' information}

Abdulbaset Hamed received his B.S. and M.Sc degrees from the Department of Electrical and Electronic Engineering, Tripoli University, Tripoli, Libya, in 1993 and 2008, respectively. Between 1998 and 2008, he held industry positions: he joined the Libyan telecommunication company as an Intelsat and Arabsat satellite earth stations engineer and was a head of the national satellite services department. He also worked with Thales Alenia Space and RascomStar companies in collaboration on the RC1 and RQ1R satellites, as a satellite control engineer. Mr. Hamed taught communication and signal processing courses at Tripoli University between 2009 and 2012. Now, he is pursuing his Ph.D at Western University, London, Canada. His research concerns wireless channel models and statistics, spectral and energy efficiency analysis, and Millimeter wave communication systems. Currently, his research is mainly focused on reducing the energy that is consumed by telecommunication networks to decrease greenhouse gases, which partially helps protect our plant. Mr. Hamed has authored and published many technical papers in international journals and conferences. He received the best paper award for two published papers at the IAENG 2015 conference. His research is administered by the Canadian Bureau for International Education. Raveendra Rao received the M.Tech. and Ph.D degrees from the Indian Institute of Technology, Delhi, India, in 1982 and 1989, respectively. From 1988 to 1999 , he was a tenured member of the Delhi Institute of Technology. He served the Institute in various capacities including Chair of the Electrical and Computer Engineering Department and Member of the Board of Governors. From 1996 to 1998, he was the Chair of Electronic Department and Deputy Principal of Caledonian Collage of Engineering (Affiliate of Glasgow Caledonian Collage of Engineering, Glasgow, UK), Muscat, Oman. Also, he was served on the engineering faculties of Delhi, Mysore, and Roorkee Universities in India. Since 1999, he has been with the Department of Electrical and Computer Engineering, University of Western Ontario, London, ON, Canada. He founded the Communication and Signal Processing (CASP) Laboratory, Delhi Institute of Technology, and was in charge of the Laboratory from 1989 to 1999 . He played major roles in the establishment and development of Electronic and Communication Engineering Laboratories, Caledonian Collage of Engineering. He is the founding member of Innovation Centre of Information Engineering (ICIE) at UWO, London, Canada. His research interests include digital communications, wireless networks, statistical signal processing, and multicarrier and multiuser communication systems. He has authored more than 50 technical papers in journals and international conferences. He has supervised more than 30 M.S. and PhD. students. Dr. Rao received the IEEE AES/COM Chapter India Award in 1985 for contributions in the area of communications, the Young Scientist of International Union of Radio Science (URSI) award in 1987, and the Student Journal Award of IETE (India) for the best paper published in 1994. He was nominated for the prestigious Vikas Jyothi Award, India, in 1993. He is a Fellow of the IETE and a registered Professional Engineer in the Province of Ontario, Canada. He is listed in Asia's Who's Who of Men and Women of Achievement.

\section{Competing interests}

The authors declare that they have no competing interests.

\section{Publisher's Note}

Springer Nature remains neutral with regard to jurisdictional claims in published maps and institutional affiliations.

Received: 11 October 2017 Accepted: 18 April 2018 Published online: 10 May 2018

\section{References}

1. L David, An operator's view on green radio (2009)

2. A Fehske, G Fettweis, J Malmodin, G Biczok, The global footprint of mobile communications: the ecological and economic perspective. IEEE Commun. Mag. 49(8), 55-62 (2011)

3. DC Kilper, G Atkinson, SK Korotky, S Goyal, P Vetter, D Suvakovic, O Blume, Power trends in communication networks. IEEE J. Sel. Top. Quant. Electron. 17(2), 275-284 (2011)

4. ElA, in Energy Information Administration: Official Energy Statistics from the U.S. Government. Summer net internal demand, capacity resources, and capacity margins, 2012 actual, 2013-2017 projected, (2015)

5. TREND, Towards real energy-efficient network design, european network of excellence (2017). Available: http://www.fp7-trend.eu/content/links. Accessed 6 Apr 2017 and 20 May 2017

6. Y Chen, S Zhang, S Xu, GY Li, Fundamental trade-offs on green wireless networks. IEEE Commun. Mag. 49(6), 30-37 (2011)

7. A Hamed, M Alsharef, RK Rao, Analysis of energy and spectral efficiency in urban shadowing environment. IAENG Int. J. Comput. Sci. 43(2), 237-244 (2016)

8. AM Hamed, A Noorwali, RK Rao, in Electrical and Computer Engineering (CCECE), 2016 IEEE Canadian Conference on. Energy efficient adaptive transmission in generalized-k fading channel (IEEE, Vancouver, 2016), pp. 1-5

9. A Hamed, RK Rao, in Performance Evaluation of Computer and Telecommunication Systems (SPECTS), 2016 International Symposium on. Bandwidth and power efficiency analysis of fading communication link (IEEE, Montreal, 2016), pp. 1-7

10. Y Li, M Sheng, C Yang, X Wang, Energy efficiency and spectral efficiency tradeoff in interference-limited wireless networks. IEEE Commun. Lett. 17(10), 1924-1927 (2013)

11. AM Alam, P Mary, J-Y Baudais, X Lagrange, in Vehicular Technology Conference (VTC Fall), 2015 IEEE 82nd. Energy efficiency-spectral efficiency tradeoff in interference-limited wireless networks with shadowing (IEEE, Boston, 2015), pp. 1-5

12. J Akhtman, L Hanzo, in Vehicular Technology Conference Fall (VTC 2009-Fall), 2009 IEEE 70th. Power versus bandwidth-efficiency in wireless communications: The economic perspective (IEEE, Anchorage, 2009), pp. $1-5$

13. LWei, RQ Hu, Y Qian, G Wu, Energy efficiency and spectrum efficiency of multihop device-to-device communications underlaying cellular networks. IEEE Trans. Veh. Technol. 65(1), 367-380 (2016)

14. AM Hamed, RK Rao, in Electrical and Computer Engineering (CCECE), 2017 IEEE 30th Canadian Conference on. Energy and spectral efficiency in cellular networks considering fading, path loss, and interference (IEEE, Windsor, 2017), pp. 1-5

15. M-S Alouini, Goldsmith AJ, Area spectral efficiency of cellular mobile radio systems. IEEE Trans. Veh. Technol. 48(4), 1047-1066 (1999)

16. H Pervaiz, L Musavian, Q Ni, in Communication Workshop (ICCW), 2015 IEEE International Conference on. Area energy and area spectrum efficiency trade-off in $5 \mathrm{~g}$ heterogeneous networks (IEEE, London, 2015), pp. $1178-1183$

17. Y Kim, T Kwon, D Hong, Area spectral efficiency of shared spectrum hierarchical cell structure networks. IEEE Trans. Veh. Technol. 59(8), 4145-4151 (2010)

18. L Zhang, H-C Yang, Generalized area spectral efficiency: an effective performance metric for green wireless communications. IEEE Trans. Commun. 62(2), 747-757 (2014), Hasna, MO

19. D Tsilimantos, J-M Gorce, K Jaffrès-Runser, HV Poor, Spectral and energy efficiency trade-offs in cellular networks. IEEE Transa. Wirel. Commun. 15(1), 54-66 (2016)

20. G Efthymoglou, P Bithas, A Kanatas, Exact SNR and SIR analysis in poisson wireless networks.Electron. Lett. 53(5), 356-358 (2017)

21. F Babich, F Vatta, Effects of sectorization on cellular radio systems capacity with different traffic loads. Wirel. Pers. Commun. 21(3), 269-288 (2002) 
22. IS Gradshteyn, IM Ryzhik, Table of integrals, series, and products. (Academic press, Burlington, 2014)

23. WR Inc, The wolfram functions site for special mathematical functions (2017). Available: http://functions.wolfram.com

24. EV Belmea, S Lasaulce, M Debbah, in Personal, Indoor and Mobile Radio Communications Workshops (PIMRC Workshops), 2010 IEEE 21st International Symposium on. A survey on energy-efficient communications (IEEE, Instanbul, 2010), pp. 289-294

25. S Khakurel, L Musavian, T Le-Ngoc, in Vehicular Technology Conference (VTC Spring), 2013 IEEE 77th. Trade-off between spectral and energy efficiencies in a fading communication link (IEEE, Dresden, 2013), pp. 1-5

26. Y A Prudnikov, O Brychkov, Marichev, Integrals and series. vol. 3, Zb/1103, vol. 33300. (Gordon and Breach Science Publishers, New York, 1990)

27. J Peng, P Hong, KXue, Energy-aware cellular deployment strategy under coverage performance constraints. IEEE Trans. Wirel. Commun. 14(1), 69-80 (2015)

28. J Chen, L-C Wang, C-H Liu, in Personal, Indoor, and Mobile Radio Communication (PIMRC), 2014 IEEE 25th Annual International Symposium on. Coverage probability of small cell networks with composite fading and shadowing (IEEE, Washington, 2014), pp. 1965-1969

\section{Submit your manuscript to a SpringerOpen ${ }^{\circ}$ journal and benefit from:}

- Convenient online submission

Rigorous peer review

- Open access: articles freely available online

- High visibility within the field

- Retaining the copyright to your article

Submit your next manuscript at $>$ springeropen.com 